\title{
Chemical modification of styrene-butadiene-styrene (SBS) rubber by reactive grafting with maleic anhydride
}

\author{
V. L. LASALLE ${ }^{1}$, M. D. FAILLA ${ }^{1}$, ENRIQUE M. VALLÉS ${ }^{1}$, \\ CARMEN M. CEPEDA-JIMÉNEZ ${ }^{2}$, ROSA TORREGROSA-MACIÁ ${ }^{2}$ \\ and JOSÉ MIGUEL MARTÍN-MARTÍNEZ ${ }^{2, *}$ \\ ${ }^{1}$ PLAPIQUI, Planta Piloto de Ingeniería Química, UNS/CONICET, \\ C.C 717-8000 Bahía Blanca, Argentina \\ ${ }^{2}$ Adhesion and Adhesives Laboratory, Department of Inorganic Chemistry, University of Alicante, \\ 03080 Alicante, Spain
}

Received in final form 14 June 2003

\begin{abstract}
A procedure to increase the adhesion of block styrene-butadiene-styrene (SBS) rubber consisting of the reactive grafting with maleic anhydride (MA) in the presence of an organic peroxide radical initiator is proposed. The influence of the reactive grafting on the surface properties of SBS has been studied with special emphasis on the improvement of the adhesion to polyurethane adhesive. The grafting of MA onto SBS was carried out in the presence of different concentrations of 2,5-dimethyl-2,5-di(tertbutyl peroxy) hexane (DBPH) as initiator to generate oxygen radicals by thermal decomposition, which induce the grafting reaction. The modification process was performed in the molten state using a Brabender mixer to premix the reactants and a hot press to initiate the functionalizing reaction. ATR-IR and XPS spectroscopies were employed to verify the grafting of MA on SBS. The changes in wettability on the modified SBS rubber were determined by contact angle measurements. Adhesion properties were evaluated from T-peel tests of SBS rubber/polyurethane adhesive joints. Reasonable extents of MA grafting on SBS were obtained (evidenced by the presence of a weak carbonyl vibration at $1700 \mathrm{~cm}^{-1}$ in the ATR-IR spectra and by the carbon-oxygen band at a binding energy of $287.0 \mathrm{eV}$ in the XPS spectra). The higher the DBPH amount, the higher the MA amount grafted onto the SBS surface. The maximum grafting level was obtained using $2 \mathrm{wt} \%$ MA. Grafted species seemed to be mainly concentrated on the surface of the SBS-molded sheets. The wettability of the modified rubber increased with respect to the original polymer, new carbon-oxygen moieties were created and the $\mathrm{C} / \mathrm{O}$ ratio increased. A noticeable enhancement in peel strength values was observed, which was ascribed to the creation of interfacial interactions between the polyurethane and the SBS rubber surfaces.
\end{abstract}

Keywords: Grafting; maleic anhydride; rubber; surface characterization;XPS; ATR-IR spectroscopy.

*To whom correspondence should be addressed. Phone: (34-96) 590-3977. Fax: (34-96) 590-3454. E-mail: jm.martin@ua.es 


\section{INTRODUCTION}

The chemical modification of commercial polymers has become a common approach to produce novel materials with improved properties [1]. One of the most useful methods of modification is the grafting of polar groups onto polymer chains. Several reactants containing different polar groups have been used for this purpose. Short-chain unsaturated polycarboxylic acid, nitriles, amides, anhydrides and esters are some of the reactants more frequently used for the functionalization of polyolefins. The grafting of functional groups onto the polymer chains is promoted by addition of organic peroxides. The thermal decomposition of the $\mathrm{RO}-\mathrm{OR}^{\prime}$ bond induces the formation of $\mathrm{RO}^{\bullet}$ free radicals that attack the molecules of the polymer, subtracting hydrogen atoms and producing reactive radicals, which, in turn, react with the added functionalizing monomers.

Styrene-butadiene-styrene (SBS) is a well-known thermoplastic with a block co-polymer structure widely employed in the rubber industry. As a result of its low surface energy, the adhesion of SBS to other materials using standard adhesives requires chemical or mechanical treatment to improve bonding. In order to reach adequate adhesion, its applications in the shoe industry require physical and/or chemical modification of the SBS surfaces in order to be glued. In this context, the aim of this study was to look for simpler procedures to chemically modify SBS polymeric chains with monomers containing polar groups. This may be a useful procedure to avoid the application of surface treatment to improve the adhesion of rubber with polyurethane adhesive.

There is not enough information in the literature about grafting maleic anhydride (MA) onto SBS [2]. Gergen et al. [3] used a titration technique to quantify the degree of MA grafting induced by organic peroxides. Saito et al. [4] grafted MA onto polymeric chains without using chemical initiators. They showed that grafting reaction could be carried out by thermal effect following the so-called 'n-reaction' mechanism. Steinkamp and Grail reported similar findings [5]. They also claimed an increase in adhesion and mechanical properties of SBS. In recent studies, Sanchez Solís et al. [6] investigated the grafting of MA onto SBS to induce compatibility and improve impact resistance of SBS-PET (poly(ethylene terephthalate))-blends using benzoyl peroxide as radical initiator. They also found that the addition of adhesion promoters had a great influence on the phase dispersion and mechanical properties of these blends. Furthermore, different researchers [7-10] showed that MA was grafted onto SBS only by removal of an allylic hydrogen atom.

In this paper, some preliminary results are presented to demonstrate the feasibility of reactive grafting of SBS with MA to improve the adhesion of SBS rubber to polyurethane adhesives. 


\section{EXPERIMENTAL}

\subsection{Materials}

Radial block co-polymer styrene-butadiene-styrene (Solprene 416, Repsol Química, Santander, Spain) was used in this study. It has a density of $0.94 \mathrm{~g} / \mathrm{cm}^{3}$ (ASTM D-297), an intrinsic viscosity of 2.2 Pa (ASTM D-2857), and a styrene block content of 30\%. 2,5-dimethyl 2,5-di(tertbutyl peroxy) hexane (DBPH) from Azko Nobel Chemicals was used as radical initiator. Maleic anhydride was provided by Anedra (Buenos Aires, Argentina).

\subsection{Grafting procedure}

Batches of $35 \mathrm{~g}$ each were prepared by premixing all the reactants (MA, DBPH and SBS) in a Brabender Plasticorder Mixer at $40 \mathrm{rpm}$ and $120^{\circ} \mathrm{C}$ for $5 \mathrm{~min}$. After mixing, the grafting reaction was carried in an hydraulic press. The temperature was fixed at $190^{\circ} \mathrm{C}$ and the reaction time was $20 \mathrm{~min}$. With this procedure, molded sheets of grafted SBS, $150 \times 150 \times 3 \mathrm{~mm}$ in size, were obtained.

To assure that the non-grafted MA onto the SBS rubber was removed, $1 \mathrm{~g}$ samples were dissolved in $100 \mathrm{ml}$ toluene at boiling temperature and then precipitated in $1000 \mathrm{ml}$ cold acetone. The resulting material was dried at room temperature under vacuum for $36 \mathrm{~h}$. Cast sheets of grafted SBS were wiped with isopropanol before carrying out the experimental measurements.

\subsection{Characterisation of the grafted $S B S$}

2.3.1. ATR-IR spectroscopy. About $5 \mu \mathrm{m}$ depth of the grafted SBS sheets were examined by FT-IR in a Nicolet 205 spectrometer employing the attenuated total reflectance (ATR) technique. A KRS-5 (thallium bromoiodide) crystal was used. ATR-IR spectra were recorded from 4000 to $400 \mathrm{~cm}^{-1}$ with an incidence angle of $45^{\circ} .200$ scans were averaged for each spectrum with $4 \mathrm{~cm}^{-1}$ resolution. Similar studies were carried out on bulk-modified SBS after its surface layer had been removed.

2.3.2. X-ray photoelectron spectroscopy (XPS). The original and modified SBS surfaces were analyzed with a VG Scientific Microtech Multilab spectrometer, with an $\mathrm{Mg} \mathrm{K}_{\alpha} \mathrm{X}$-ray source $(1253.6 \mathrm{eV})$ operating at $15 \mathrm{keV}$ and $300 \mathrm{~W}$. The angle of incidence of the X-rays on the sample was set to $45^{\circ}$. Prior to analysis, samples were outgassed in a vacuum chamber to a pressure lower than $5 \times 10^{-8}$ torr. The analysis was performed on $5 \times 2 \mathrm{~mm}$ rubber surfaces. For each sample, a survey scan encompassing the region $0-1200 \mathrm{eV}$ was first obtained. More detailed scannings of all observed photopeaks in the survey scan were carried out in the $20 \mathrm{eV}$ range. Binding energies of all photopeaks were referenced to the $\mathrm{C}_{1 \mathrm{~s}}$ photopeak position for $\mathrm{C}-\mathrm{C}$ and $\mathrm{C}-\mathrm{H}$ (hydrocarbons) species at $285.0 \mathrm{eV}$. Atomic concentration calculations were carried out using a VGX900-W system. 
2.3.3. Contact angle measurements. The differences in the surface properties of grafted SBS with respect to original (non-grafted) rubber were evaluated by this technique. A drop of the test liquid $\left(\mathrm{CH}_{2} \mathrm{I}_{2}\right)$, approx. $4 \mu \mathrm{l}$, was placed on the SBS rubber surface with a syringe. The SBS sample was maintained in an environmental chamber saturated with the vapour of the test liquid. The contact angle measurements were made after 15 min of drop deposition to ensure equilibrium conditions. The reported measurements were an average of six determinations taken in different areas of the surface.

2.3.4. T-peel tests. T-peel tests were performed on adhesive joints obtained using polyurethane adhesive and specimens of the original and modified SBS rubber. The polyurethane adhesive was prepared by dissolving $15 \mathrm{wt} \%$ of commercial polyurethane pellets (Desmocoll 540 provided by Bayer, Leverkusen, Germany) in MEK (methyl ethyl ketone). A Cowles mechanical stirrer operating at $1000 \mathrm{rpm}$ was used for this purpose. The dissolution of the polyurethane was completed after stirring for $2 \mathrm{~h}$. This adhesive solution was employed to join two similarly modified rectangular strips of SBS rubber $3 \times 10 \times 150 \mathrm{~mm}$ in size. About $1 \mathrm{ml}$ of adhesive solution was applied on each SBS strip. A 30-min period under open air was allowed to facilitate solvent evaporation and ensure uniform rubber wetting. Then, the solid polyurethane films on the SBS surfaces were reactivated using an infrared lamp at $90-100^{\circ} \mathrm{C}$ for $24 \mathrm{~s}$, and the molten polyurethane films were bonded for $11 \mathrm{~s}$ at a pressure of $0.8 \mathrm{MPa}$. The adhesive joints were maintained at room temperature $\left(23^{\circ} \mathrm{C}\right)$ under a relative humidity of $50 \%$ for periods of $15 \mathrm{~min}$ and $72 \mathrm{~h}$. At the end of these periods, peel tests were carried out using an Instron 4411 machine with a pull rate of $100 \mathrm{~mm} / \mathrm{min}$. The peel strength values reported in this work are the average of three measurements with each SBS-modified sample.

\section{RESULTS AND DISCUSSION}

Figure 1 compares the ATR-IR spectra in the region between $1500-2000 \mathrm{~cm}^{-1}$ of a virgin SBS sample, a grafted unpurified sample (containing all the added MA), the surface of which was only washed with acetone to remove organic contaminants during processing and manipulation, and a purified grafted SBS sample obtained by treating the grafted unpurified SBS with hot toluene (thus containing only the grafted MA because the residual non-grafted MA was removed). The ATRIR spectra show that the reaction of SBS polymer chains with 2 wt\% MA and $0.05 \mathrm{wt} \%$ DBPH grafts successfully a reasonable small amount of MA onto the SBS. The precise amount of MA grafted onto SBS was not determined in this study. Although several procedures to establish the grafting degree of MA in polymers have been proposed $[9,10]$, none of them provides adequate results. In fact, the ATR-IR spectrum of the virgin $\mathrm{SBS}$ does not show any $\mathrm{C}=\mathrm{O}$ vibration, whereas the unpurified grafted SBS shows a sharp band due to carboxylic acid $\left(1710 \mathrm{~cm}^{-1}\right)$ which indicates the presence of both non-grafted and grafted MA onto the SBS 


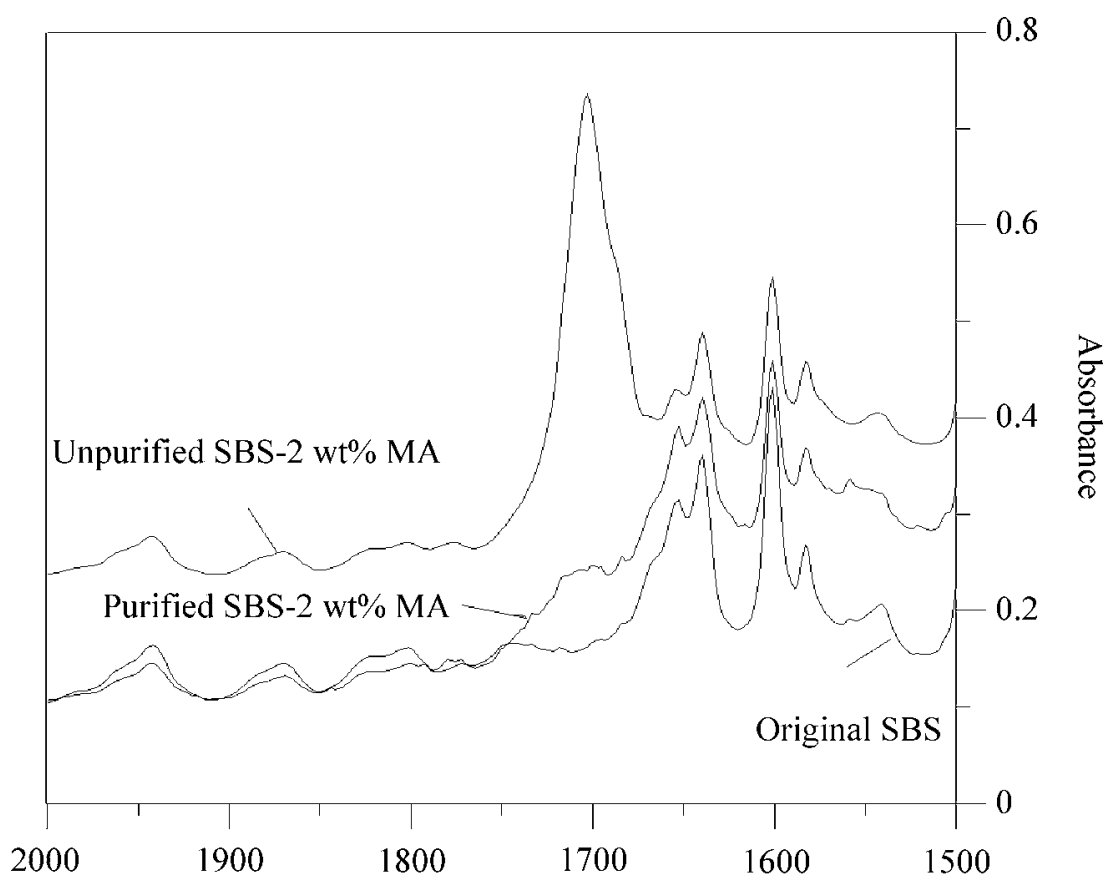

Wavenumber $\left(\mathrm{cm}^{-1}\right)$

Figure 1. ATR-IR spectra of original, and unpurified (containing all MA) and purified (containing MA grafted only) SBS rubber. $0.050 \mathrm{wt} \% \mathrm{DBPH}$ was added as radical initiator.

chains. The band location, slightly shifted from the absorption bands of the typical carbonyl absorption of the anhydride $\left(1730-1790 \mathrm{~cm}^{-1}\right)$, indicates that probably the MA has been hydrolysed during the mixing or reaction process. These findings are in agreement with recent results by Aimin and Chao [10]. As result of removal of the non-grafted MA on the SBS with hot toluene, the $\mathrm{C}=\mathrm{O}$ band intensity at $1710 \mathrm{~cm}^{-1}$ diminishes with respect to that of the unpurified rubber because the unreacted MA is removed not only from the surface but also from the bulk polymer, and only the grafted MA remains. Very similar results were obtained with the SBS samples modified with different concentrations of DBPH and MA.

Figure 2 compares the surface (Fig. 2a) and bulk (Fig. 2b) ATR-IR spectra of the unpurified SBS rubber modified with different amounts of MA. The ATR-IR spectra of the bulk were obtained by removing the external surface of the SBS rubber using a surgical blade. In the bulk ATR-IR spectra the bands associated with the $\mathrm{C}=\mathrm{O}$ bond are not present in detectable amounts. This is an indication that the MA moieties in the SBS rubber tend to concentrate on its surface.

XPS technique provides further evidence of reactive grafting of MA on the SBS. Tables 1 and 2 show the elemental composition of the modified SBS surfaces before and after purification with hot toluene, respectively. The presence of silicon reveals 
MA grafted-SBS (external surface)

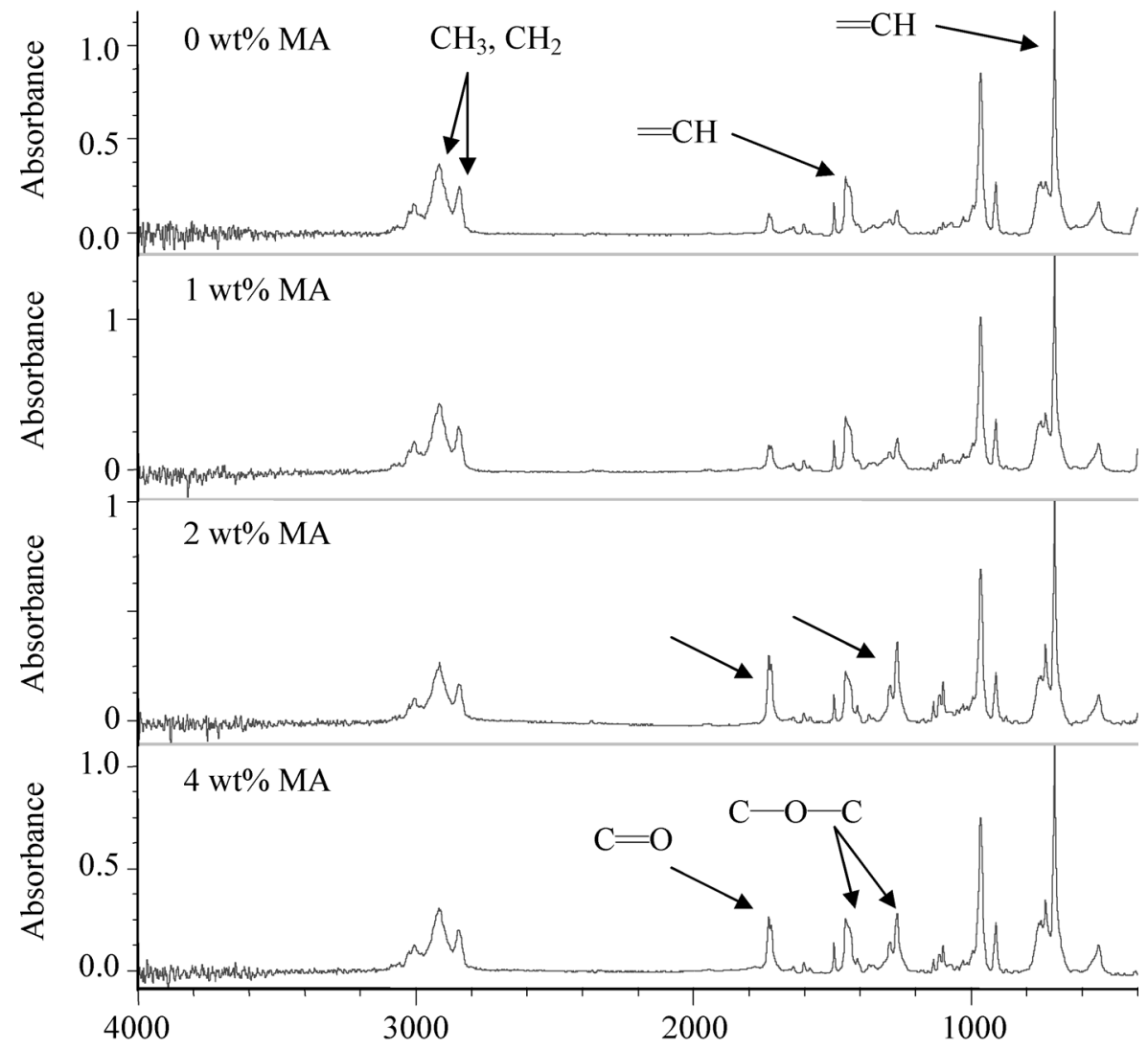

Wavenumber $\left(\mathrm{cm}^{-1}\right)$

(a)

Figure 2. ATR-IR spectra of modified SBS rubber with different amounts of MA and 0.050 wt $\%$ DBPH. (a) Surface; (b) after removal of the uppermost surface layer.

Table 1.

XPS elemental composition (at\%) of unpurified SBS rubber containing different amounts of MA and $0.050 \mathrm{wt} \% \mathrm{DBPH}$

\begin{tabular}{lllll}
\hline Element & $0 \mathrm{wt} \% \mathrm{MA}$ & $1 \mathrm{wt} \% \mathrm{MA}$ & $2 \mathrm{wt} \% \mathrm{MA}$ & $4 \mathrm{wt} \% \mathrm{MA}$ \\
\hline $\mathrm{C}$ & 87.0 & 89.6 & 84.0 & 87.4 \\
$\mathrm{O}$ & 9.2 & 8.5 & 13.3 & 10.5 \\
$\mathrm{~N}$ & 0.3 & 0.4 & 0.4 & 0.2 \\
$\mathrm{Si}$ & 3.4 & 1.6 & 2.4 & 1.9 \\
$\mathrm{O} / \mathrm{C}$ & 0.11 & 0.09 & 0.16 & 0.12 \\
\hline
\end{tabular}


MA grafted-SBS (bulk)

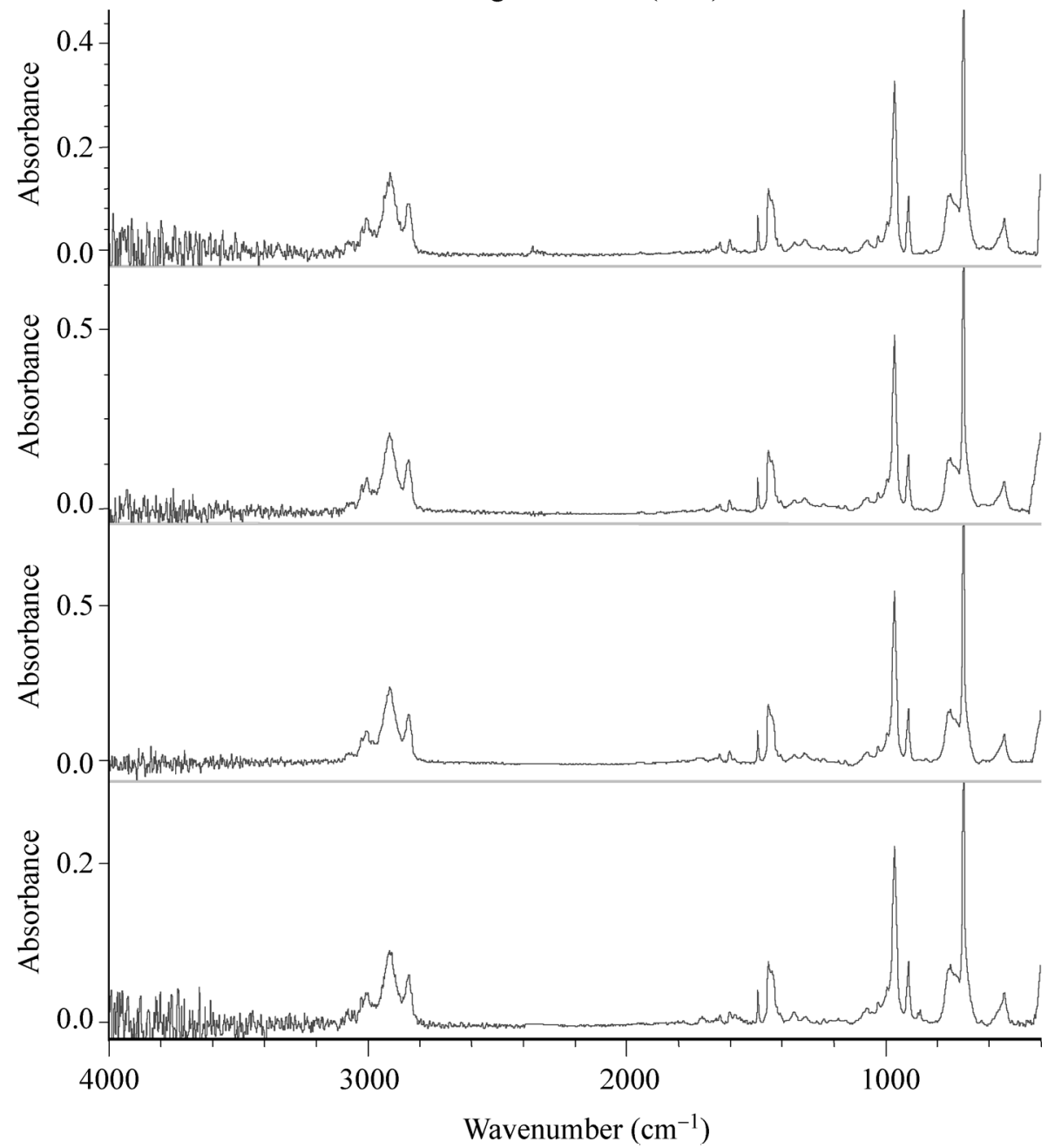

(b)

Figure 2. (Continued).

that some silica contamination of the samples during processing was present. This complicates the interpretation of the data because silica also contains oxygen atoms (two oxygen atoms for each silicon atom). If a correction for the oxygen present in these impurities is performed (Table 3), all the rubber samples containing MA show higher oxygen content than the untreated SBS. After purification with hot toluene, the carbon content increases and the oxygen content decreases (i.e. the $\mathrm{O} / \mathrm{C}$ ratio decreases) indicating the removal of unreacted MA. As the amount of MA added to the rubber is increased from 1 to $4 \mathrm{wt} \%$ the decrease in oxygen content observed after purification becomes more significant. Whereas the unpurified SBS 
Table 2.

XPS elemental composition (at $\%$ ) of purified SBS rubber containing different amounts of MA and $0.050 \mathrm{wt} \% \mathrm{DBPH}$

\begin{tabular}{lcccc}
\hline Element & 0 wt\% MA & 1 wt\% MA & 2 wt\% MA & 4 wt\% MA \\
\hline $\mathrm{C}$ & 87.0 & 90.6 & 84.4 & 89.7 \\
$\mathrm{O}$ & 9.2 & 7.5 & 11.4 & 6.8 \\
$\mathrm{~N}$ & 0.3 & 0.7 & 0.3 & 0.3 \\
$\mathrm{Si}$ & 3.4 & 1.2 & 3.8 & 3.1 \\
$\mathrm{O} / \mathrm{C}$ & 0.11 & 0.08 & 0.14 & 0.08 \\
\hline
\end{tabular}

Table 3.

Corrected* XPS elemental composition(at\%) of purified SBS rubber containing different amounts of MA and $0.050 \mathrm{wt} \% \mathrm{DBPH}$

\begin{tabular}{lllll}
\hline Element & $0 \mathrm{wt} \% \mathrm{MA}$ & $1 \mathrm{wt} \% \mathrm{MA}$ & $2 \mathrm{wt} \% \mathrm{MA}$ & $4 \mathrm{wt} \% \mathrm{MA}$ \\
\hline $\mathrm{C}$ & 97.0 & 94.0 & 95.4 & 96.0 \\
$\mathrm{O}$ & 2.7 & 5.3 & 4.3 & 3.6 \\
$\mathrm{~N}$ & 0.3 & 0.7 & 0.3 & 0.4 \\
$\mathrm{O} / \mathrm{C}$ & 0.03 & 0.06 & 0.05 & 0.04 \\
\hline
\end{tabular}

* Percentages of elements were corrected by substracting silicon and the corresponding amount of oxygen (a stoichiometry of $\mathrm{SiO}_{2}$ was assumend).

Table 4.

$\mathrm{CH}_{2} \mathrm{I}_{2}$ contact angles (degrees) on SBS rubber grafted with different amounts of MA and $0.050 \mathrm{wt} \% \mathrm{DBPH}$

\begin{tabular}{ll}
\hline MA (wt \%) & $\theta\left(\mathrm{CH}_{2} \mathrm{I}_{2}\right)$ \\
\hline 0 & 50 \\
1 & 33 \\
2 & 39 \\
4 & 40 \\
\hline
\end{tabular}

containing $1 \mathrm{wt} \%$ MA has an oxygen content of 8.5 at $\%$ and it decreases to 7.5 at $\%$ after purification, for the SBS containing $4 \mathrm{wt} \%$ MA the oxygen content decreases from 10.5 at\% to 6.8 at\%. This is an indication that the amount of MA grafted on the rubber chains is limited to a certain value and higher amounts of MA do not necessarily produce higher degree of grafting of the rubber.

Because the grafting of MA seems to be mainly restricted to the SBS surface, the wettability of the modified SBS rubbers was determined using contact angle measurements. Equilibrium contact angles on virgin and functionalized rubbers with MA are shown in Table 4. The $\mathrm{CH}_{2} \mathrm{I}_{2}$ contact angle values on grafted SBS decreases, indicating an improved wettability of SBS rubber after grafting. 
The data provided by ATR-IR spectroscopy correspond to about $5 \mu \mathrm{m}$ depth on the SBS surface. Therefore, XPS was used to obtain information on the chemistry in the outermost SBS surface (about $5 \mathrm{~nm}$ ). Table 2 shows the chemical composition of the purified SBS rubber containing different amounts of MA and $0.050 \mathrm{wt} \%$ $\mathrm{DBPH}$. Grafting with MA produces an increase in $\mathrm{O} / \mathrm{C}$ ratio, which is an indication of the reaction of MA with the rubber chains. The highest oxygen content is seen for the SBS rubber grafted with $2 \mathrm{wt} \%$ MA. Silicon is also present on the grafted rubber surfaces as a contaminant and a tiny amount of nitrogen also appears (likely due to impurities during processing of SBS rubber). The amount of DBPH also determines the grafting degree of MA onto the rubber chains. Tables 2 and 5 show that the increasing amount of DBPH favours the grafting of $2 \mathrm{wt} \%$ MA (i.e. higher amounts of oxygen and lower amounts of carbon are obtained), and this effect is less marked for the SBS rubber grafted with $4 \mathrm{wt} \%$ MA.

Figure 3 shows typical examples of curve fitting of $\mathrm{C}_{1 \mathrm{~s}}$ photopeaks for unpurified and purified SBS rubbers containing $2 \mathrm{wt} \%$ MA. Table 5 shows the percentages of carbon species for all unpurified and purified SBS rubbers. The curve fitting of unpurified grafted SBS shows the existence of carboxyl groups (binding energy = $289.4 \mathrm{eV}$ ) due to the hydrolysis of the MA duting processing. Because the maleic acid is not grafted onto the SBS rubber, these groups disappear after purification in hot toluene (Fig. 3). Furthermore, a new band due to new carbon-oxygen groups at a binding energy of $287.0 \mathrm{eV}$ appears in both unpurified and purified grafted SBS. Typical carbonyl band appears at $288.0 \mathrm{eV}$ but the presence of a chemical environment less electronegative produces displacement of the binding energy to lower values [11]. The grafting of MA onto the polymer chains allows the formation of carbonyl-type groups which seems to be displaced to lower binding energy. In fact, purification of grafted SBS maintains the band at $287.0 \mathrm{eV}$, and the percentages are relatively similar in both unpurified and purified samples (Table 5). On the other hand, Table 5 shows higher percentages of carbon-oxygen moieties by increasing the amount of MA up to $2 \mathrm{wt} \%$, although for the $4 \mathrm{wt} \%$ MA grafted $\mathrm{SBS}$, a reduction in carbon-oxygen groups is obtained, probably due to the lower degree of grafting. Finally, the lower amount of DBPH (i.e. $0.025 \mathrm{wt} \%$ ) produces less degree of grafting.

The modifications in both surface chemistry and wettability in the SBS containing MA affect the peel strength values of SBS/polyurethane adhesive joints. Figure 4 shows that the initial peel strength value (obtained $15 \mathrm{~min}$ after joint formation) of virgin SBS/polyurethane adhesive joint is of the order of $300 \mathrm{~N} / \mathrm{m}$, this value increases to about $500 \mathrm{~N} / \mathrm{m}$ after $72 \mathrm{~h}$ (final peel strength) due to polyurethane crystallization. These peel strength values are considered to be low, which means that virgin SBS exhibits poor adhesion properties to polyurethane adhesives. The improvement in adhesion properties by reactive grafting of SBS rubber with MA is significant. The peel strength values increase in the joints produced with SBS containing MA is more marked by increasing the MA content up to $2 \mathrm{wt} \%$. This is in agreement with the variations in surface chemistry in the grafted SBS rubbers 


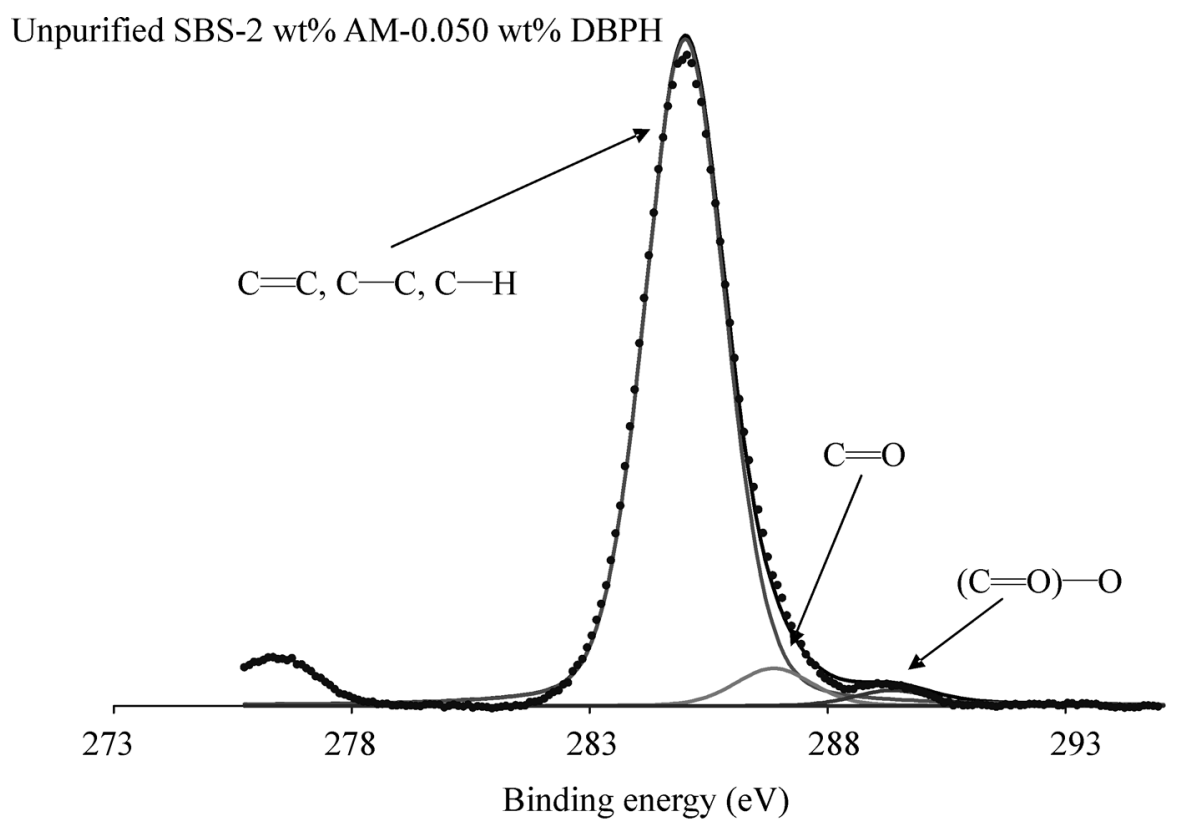

Purified SBS-2 wt\% AM-0.050 wt\% DBPH

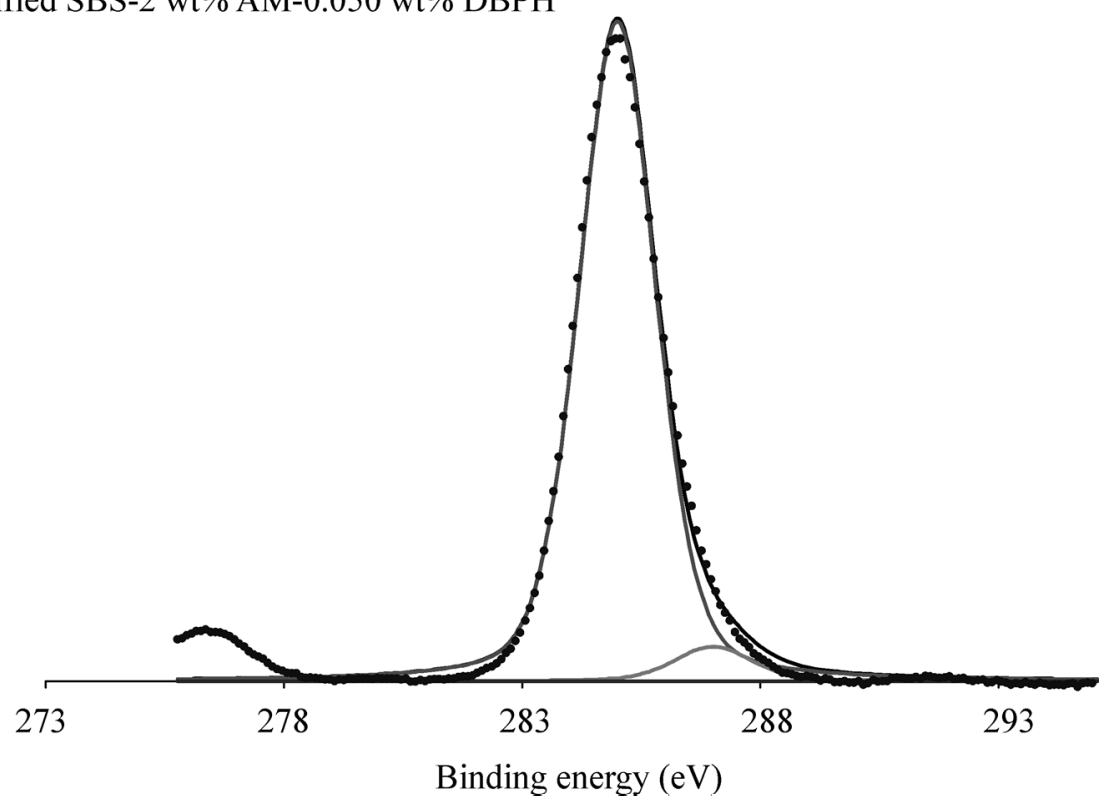

Figure 3. XPS $\mathrm{C}_{1 \mathrm{~s}}$ curve fitting of unpurified (containing all MA) and purified (containing grafted MA only) SBS rubber containing $2 \mathrm{wt} \% \mathrm{MA}$ and $0.050 \mathrm{wt} \%$ DBPH. $0.025 \mathrm{wt} \% \mathrm{DBPH}$. 
Table 5.

XPS at $\%$ of species obtained from $\mathrm{C}_{1 \mathrm{~s}}$ curve fitting of unpurified and purified SBS rubber containing different amounts of MA and DBPH

\begin{tabular}{|c|c|c|c|c|c|c|}
\hline \multirow[t]{2}{*}{ MA (wt\%) } & \multirow[t]{2}{*}{ DBPH (wt\%) } & \multicolumn{3}{|c|}{ Unpurified } & \multicolumn{2}{|l|}{ Purified } \\
\hline & & $285.0 \mathrm{eV}$ & $287.0 \mathrm{eV}$ & $289.4 \mathrm{eV}$ & $285.0 \mathrm{eV}$ & $287.0 \mathrm{eV}$ \\
\hline 0 & - & 96.5 & 3.5 & - & 96.5 & 3.5 \\
\hline 1 & 0.050 & 97.1 & 2.3 & 0.6 & 97.3 & 2.7 \\
\hline 2 & 0.050 & 91.7 & 5.7 & 2.6 & 95.0 & 5.0 \\
\hline 4 & 0.050 & 96.8 & 2.2 & 1.1 & 97.8 & 2.2 \\
\hline 2 & 0.025 & 97.4 & 2.6 & - & 97.5 & 2.5 \\
\hline 4 & 0.025 & 98.3 & 1.7 & - & 97.9 & 2.1 \\
\hline
\end{tabular}

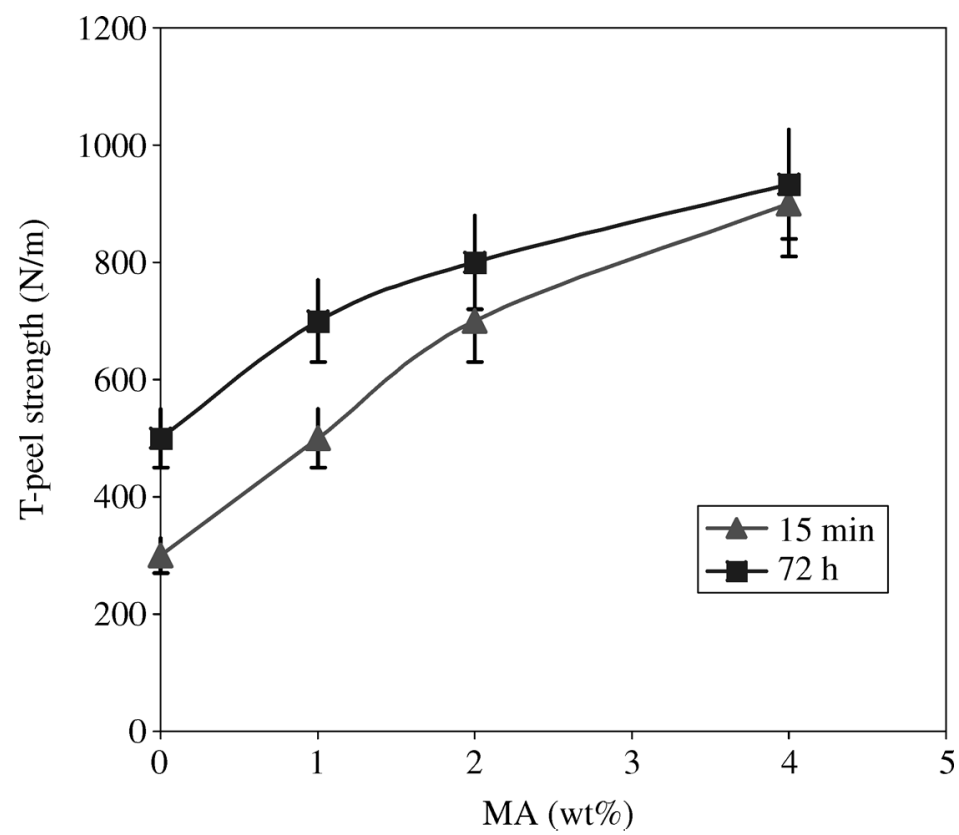

Figure 4. T-peel strength values of SBS rubber/polyurethane adhesive joints as a function of the MA content in the modified SBS $(0.050 \mathrm{wt} \% \mathrm{DBPH})$. Always an adhesion failure was obtained (visual inspection).

(Fig. 2a and Tables 2 and 5). As the concentration of MA increases, the initial peel strength increases three-fold with respect to the joint produced with the virgin rubber, and the final peel strength increases by a factor of two. All peel tests show by visual inspection an adhesion failure, i.e. the failure is produced between the adhesive and the SBS rubber surface, indicating that the adhesion of SBS to polyurethane adhesive increases by reactive grafting with MA.

The improved adhesion between MA-grafted SBS rubber and the polyurethane could be due to improved interfacial interaction. ATR-IR spectroscopy was used to 


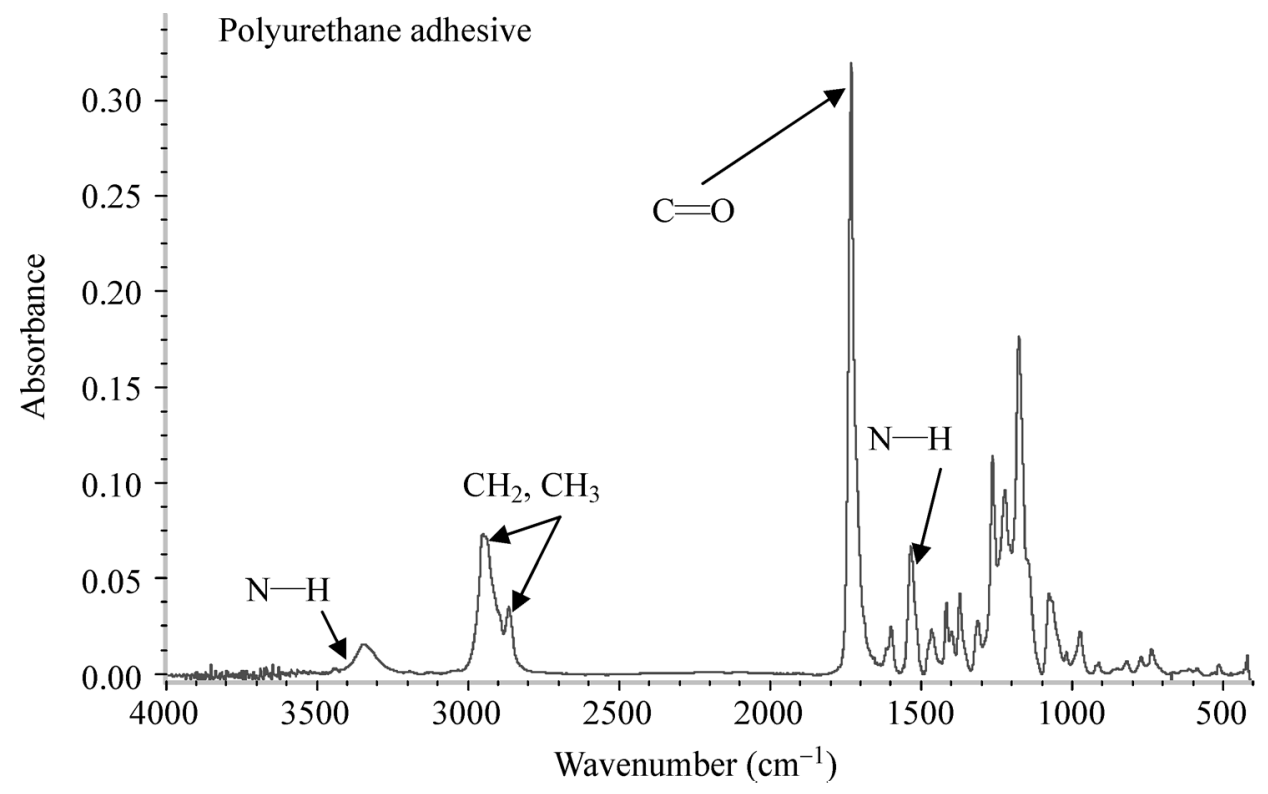

(a)

Figure 5. ATR-IR spectra of the (a) polyurethane adhesive films and (b) MA-modified SBS rubbers (4 wt $\%$ MA and $0.050 \mathrm{wt} \% \mathrm{DBPH}$ ) with thin polyurethane films on their surface. Numbers indicate the concentration of the polyurethane in the solution used to spin-coat the modified SBS rubber samples.

determine the chemical composition of thin polyurethane films deposited by spincoating of diluted adhesive solutions. The polyurethane films were obtained using solutions of the adhesive in MEK at different concentrations ranging from 0.5 to $18 \mathrm{wt} \%$, followed by drying at room temperature and reactivation at $90-100^{\circ} \mathrm{C}$ for $24 \mathrm{~s}$ (the same experimental conditions as used during joints formation). Figure 5 shows the ATR-IR spectra of the polyurethane film and of the SBS rubber modified with $4 \mathrm{wt} \%$ MA with deposited thin polyurethane films. The ATR-IR spectrum of the polyurethane film (Fig. 5a) shows typical bands corresponding to the urethane: $-\mathrm{N}-\mathrm{H}\left(1538,3347 \mathrm{~cm}^{-1}\right),-\mathrm{C}=\mathrm{O}\left(1741 \mathrm{~cm}^{-1}\right)$ and $\mathrm{C}-\mathrm{O}-\mathrm{C}(1068,1225$ $\mathrm{cm}^{-1}$ ) groups. These bands are different from those for the SBS rubber. The ATR-IR spectra corresponding to 0.5 and $1.0 \mathrm{wt} \%$ polyurethane adhesive solutions (Fig. 5b) show the disappearance of the $\mathrm{C}-\mathrm{O}-\mathrm{C}$ groups at 1216 and $1063 \mathrm{~cm}^{-1}$ and carbonyl groups at $1700 \mathrm{~cm}^{-1}$, which is indicative of some kind of interaction between these functional groups and the adhesive. Furthermore, the bands of the polyurethane cannot be distinguished, probably because some chains interdiffusion between the polyurethane and the SBS rubber surfaces takes place. In the ATR-IR spectra corresponding to higher concentrations of the adhesive, the region of the carbonyl groups is occupied by the characteristic bands of the polyurethane and the disappearance of the carbonyl groups cannot be distinguished. 


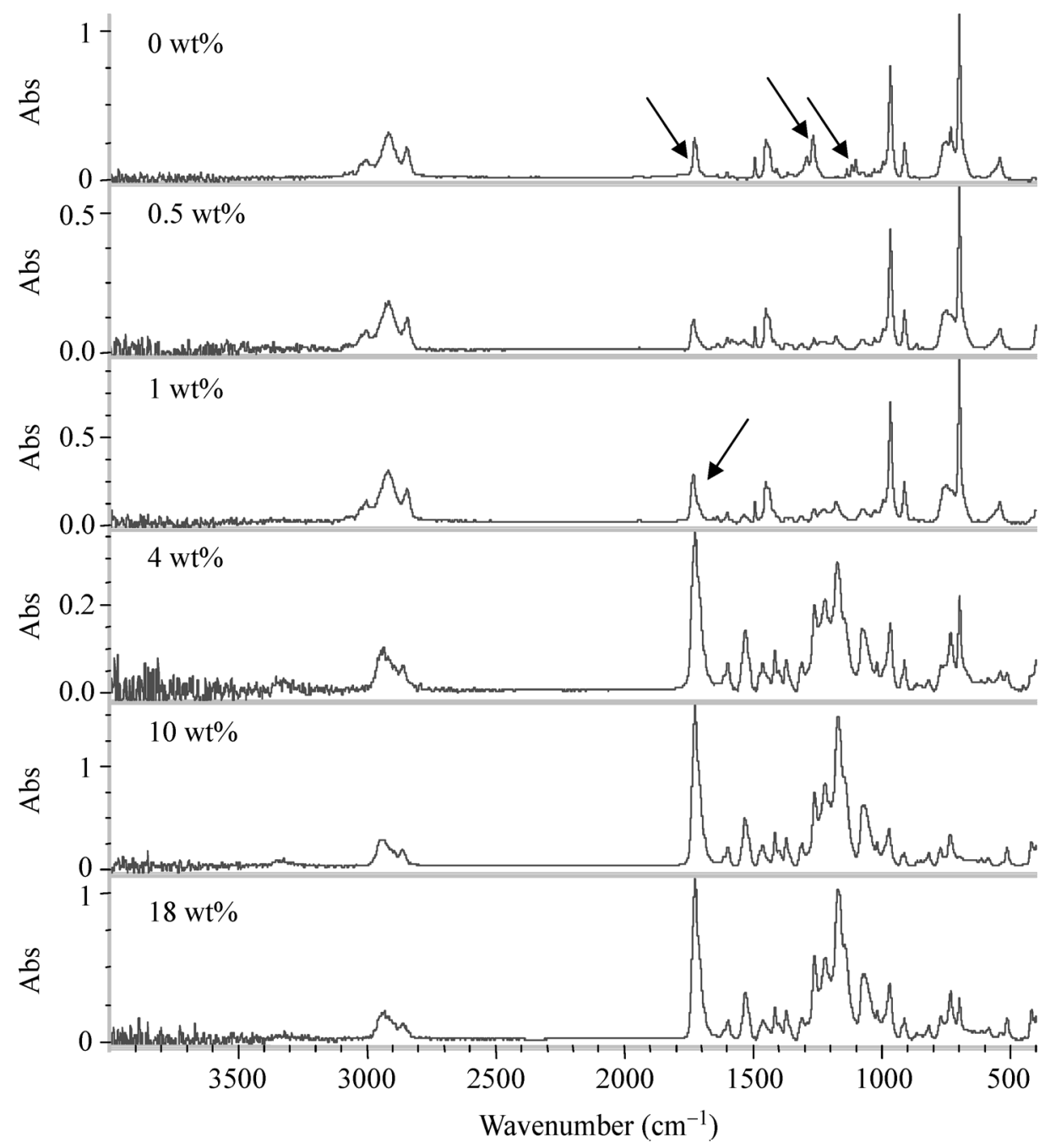

(b)

Figure 5. (Continued).

\section{CONCLUSIONS}

In this study, a procedure to increase the adhesion of SBS rubber to polyurethane adhesives is presented. This procedure consists in the incorporation of MA and a radical organic peroxide type initiator (different amounts of DBPH) during extrusion of SBS rubber. The experimental results obtained using ATR-IR and XPS spectroscopies showed that a successful grafting of MA onto the SBS polymeric chain was obtained. The higher the DBPH amount, the higher the MA amount grafted. The maximum grafting level was obtained with $2 \mathrm{wt} \%$ MA. The modified SBS rubbers possess higher wettability than the virgin rubber, carbon-oxygen moi- 
eties (binding energy $=287.0 \mathrm{eV}$ ) are created and the $\mathrm{O} / \mathrm{C}$ ratio increases. These surface modifications produced significant improvements in the peel strengths of SBS/polyurethane adhesive joints, which can be ascribed to enhanced interfacial interactions between the polyurethane and the grafted species on the SBS rubber surfaces.

\section{Acknowledgements}

The authors thank CYTED Network VIII.D for providing financial support and help in establishing research interactions between PLAPIQUI (Argentina) and the Adhesion and Adhesives Laboratory (Spain).

\section{REFERENCES}

1. M. Xanthos, in: Reactive Extrusion: Principle and Practice, J. A. Biesenberger (Ed). Polymer Processing Institute, Stevens Institute of Technology, Hoboken, NJ (1992).

2. C. Trivedi and B. M. Culbertson, in: Maleic Anhydride, p. 459. Plenum Press, New York, NY (1982).

3. N. P. Gergen, A. Lutz and T. Gelles, US Patent No. 4,578,429 (1986).

4. D. Saito, N. Asashi, I. Yamori and T. Ibaragui, US Patent No. 4,492,414(1981).

5. R. A. Steinkamp and T. J. Grail, US Patent No. 4,001,172 (1977).

6. A. Sánchez Solís, M. R. Estrada, J. Cruz and O. Manero, Polym. Eng. Sci. 40, 1216 (2000).

7. J. A. Chandrasiri and C. A. Wilkie, J. Polym. Sci. Part A: Polym. Chem. 34, 1113 (1996).

8. D. D. Jiang and C. A. Wilkie, J. Polym. Sci. Part A: Polym. Chem. 35, 965 (1997).

9. E. Passaglia, S. Ghetti, F. Picchioni and G. Ruggeri, Polymer 41, 4389 (2000).

10. Z. Aimin and L. Chao, Eur. Polym. J. 39, 1291 (2003).

11. C. D. Wagner, W. M. Riggs, L. E. Davis and J. F. Moulder, in: Handbook of X-ray Photoelectron Spectroscopy, G. E. Muilenberg (Ed.), p. 38. Perkin Elmer, Eden Prairie, MN (1979). 